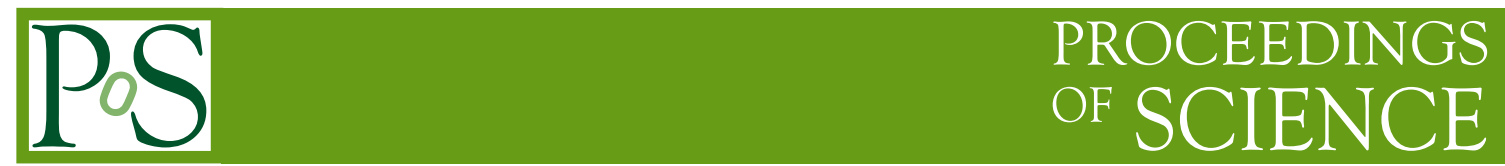

\title{
Hard hadron spectroscopy
}

\author{
Craig McNeile* \\ Department of Physics and Astronomy \\ The Kelvin Building \\ University of Glasgow \\ Glasgow G12 8QQ \\ U.K. \\ E-mail: c.mcneile@physics.gla.ac.uk
}

I review lattice QCD calculations that compute the masses of the flavour singlet pseudoscalar mesons. I describe the progress in computing the mass spectrum of light $0^{++}$mesons using lattice QCD. I also compare the results for basic quantities such as $f_{\pi}$ and $m_{\rho}$, from various different formalisms of lattice QCD. I discuss the physical value of $r_{0}$.

The XXV International Symposium on Lattice Field Theory

July 30-4 August 2007

Regensburg, Germany

${ }^{*}$ Speaker. 


\section{Introduction}

I review the status of light hadron spectroscopy from lattice QCD. I start by reviewing lattice calculations of the masses of flavour singlet pseudoscalar mesons. In the first part I will focus on lattice results for the $J^{P C}=0^{++}$scalar mesons, because these mesons are candidates for having glueball or tetraquark degrees of freedom and are still not well understood. In the second half of the talk I will compare the results for basic quantities such as the pion decay constant and mass of the light vector meson between different lattice formalisms. This is an important part of validating lattice calculations.

There are various omissions in this review. I don't include any results for baryons. Of particular note is the work of the LHPC collaboration [1] who are using a highly developed variational technique to try to fully map out the low lying baryon spectrum. Also I don't discuss any developments in the spectroscopy of mesons that include heavy quarks, although there have been many new states that have been discovered such as the $D_{s}(2317), \mathrm{X}(3872)$, and $Y(4260)$. See [2] for a review of the experiments and the results from model calculations, and [B] for a review of lattice results.

\section{The singlet pseudoscalar mesons from lattice qcd}

The large mass of the $\eta^{\prime}$ meson is thought to be caused by the QCD vacuum structure and the axial anomaly. The $\eta$ and $\eta^{\prime}$ mesons are decay products of flavour non-singlet $0^{++}$mesons, so are a natural starting point for a review with a focus on $0^{++}$mesons.

In unquenched QCD with $n_{f}=2$ sea quarks there is only one singlet pseudoscalar meson that I will use the $\eta_{2}$ notation for. The mass of the $\eta_{2}$ is expected to be around $800 \mathrm{MeV}$. The lattice calculations of the flavour singlet pseudoscalar mesons involve the computation of disconnected diagrams, that are more noisy and compute intensive, than connected correlators. With the available computing power it is possible to compute the relevant disconnected correlators. However, the intrinsic noisiness of the correlators means that many more configurations are required than for a standard lattice QCD calculation of flavour non-singlet quantities, and this makes them expensive [4, 5]. In fact the sub-groups who work on flavour singlet quantities inside a collaboration are always the people who ask for much longer simulation runs.

In figure 11 I show a plot of recent results from the ETM collaboration for the mass of the $\eta_{2}$ meson [ $\llbracket$ ]. There is an summary of the results of older lattice QCD calculations for the mass of the $\eta_{2}$ meson in [7].

Figure 11 shows that the mass of $\eta_{2}$ meson is consistent with a constant behaviour with quark mass, although the statistical errors are large. The preliminary estimate for the mass of the $\eta_{2}$, from ETMC [ [], is $\approx .88 \mathrm{GeV}\left(r_{0} m\left(\eta_{2}\right)=2\right)$.

Another complication of $2+1$ lattice QCD calculations is $\eta-\eta^{\prime}$ mixing. In lattice QCD calculations with $2+1$ flavours of sea quarks there will be mixing between light and strange interpolating operators. The experience with the last generation of $n_{f}=2$ lattice QCD calculations with pion mass at the $600 \mathrm{MeV}$ level found that the differences between quenched and unquenched QCD were mostly small [ 8$]$. This suggests that the difference between $n_{f}=2$ and $n_{f}=2+1$ will be small. However this will not be the case for the flavour singlet pseudoscalar mesons where the ground 


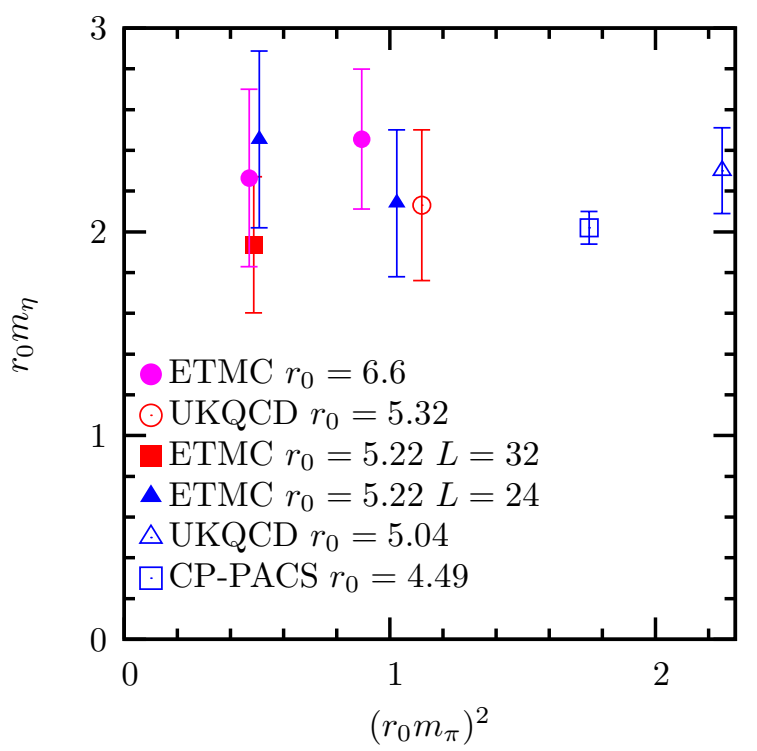

Figure 1: Summary of results for the mass of $\eta_{2}$ from calculations with light pions and $a<0.1 \mathrm{fm}$. The

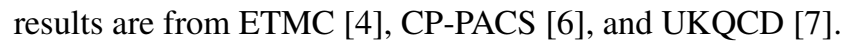

state will be the mass of the $\eta$ (mass $548 \mathrm{MeV}$ ) with $n_{f}=2+1$ flavours, but the mass of the $\eta_{2}$ (mass around $800 \mathrm{MeV}$ ) for lattice calculations with $n_{f}=2$ flavours. This will indirectly effect particles that decay via the strong interaction with a flavour singlet pseudoscalar meson as a final state, because the decay threshold will differ between $n_{f}=2$ and $n_{f}=2+1$, by the order of 250 $\mathrm{MeV}$.

Both the $\bar{u} \gamma_{5} u+\bar{d} \gamma_{5} d$ and $\bar{s} \gamma_{5} s$ will couple to the $\eta$ meson. The $\eta^{\prime}$ is the first excited state in light flavour singlet pseudoscalar channels. A modern approach to $\eta, \eta^{\prime}$ mixing is reviewed by Feldmann [9].

The CP-PACS/JLQCD collaboration used a variational technique to study the $\eta$ and $\eta^{\prime}$ meson [10]. The basis states in equation 2.1] were used to form a variational smearing matrix 2.2.

$$
\begin{aligned}
& \eta_{n}=\left(\bar{u} \gamma_{5} u+\bar{d} \gamma_{5} d\right) / \sqrt{2}, \quad \eta_{s}=\left(\bar{s} \gamma_{5} s\right), \\
& G(t)=\left(\begin{array}{ll}
\eta_{n}^{P}(t) \eta_{n}^{S}(0) & \eta_{n}^{P}(t) \eta_{s}^{S}(0) \\
\eta_{s}^{P}(t) \eta_{n}^{S}(0) & \eta_{s}^{P}(t) \eta_{s}^{S}(0)
\end{array}\right),
\end{aligned}
$$

The preliminary results presented at lattice 2006 [10]. from a lattice calculation with $a \sim 0.12$ fm, $m_{V} / m_{P S} 0.61$ to 0.78 were $m_{\eta}=0.55(2) \mathrm{GeV}$, and $m_{\eta^{\prime}}=0.87(5) \mathrm{GeV}$ (compare to with experiment, $m_{\eta}=0.548 \mathrm{GeV}$, and $m_{\eta^{\prime}}=0.958 \mathrm{GeV}$ There has also been a recent attempt to compute the masses of the $\eta, \eta^{\prime}$ mesons using improved staggered fermions [5], that is also regarded as an important theoretical test of the staggered formalism [11].

\section{The light scalar mesons from lattice QCD}

The interpretation of many $0^{++}$mesons in terms of quarks and glue degrees of freedom is 


\begin{tabular}{|c|c|c|c|c|c|}
\hline Meson & $\mathrm{I}$ & $\mathrm{M} \mathrm{MeV}$ & $\Gamma \mathrm{MeV}$ & main decay & comment \\
\hline$a_{0}(980)$ & 1 & 985 & $50-100$ & $\eta \pi$ & tetraquark, molecule, $\bar{q} q$ \\
$a_{0}(1450)$ & 1 & 1474 & 265 & $\eta \pi$ & $\bar{q} q$ \\
$\kappa$ & $1 / 2$ & 660 & 560 & $K \pi$ & not in PDG (yet) \\
$K_{0}(1430)$ & $1 / 2$ & 1414 & 290 & $K \pi$ & $\bar{q} s$ \\
\hline
\end{tabular}

Table 1: Light $0^{++}$and $0^{+}$flavour non-singlet mesons

\begin{tabular}{|c|c|c|}
\hline Group & $n_{f}$ & $m_{K_{0}} \mathrm{GeV}$ \\
\hline Prelovsek et al. [16] & 2 & $1.6 \pm 0.2$ \\
McNeile and Michael [17] & 2 & $1.1-1.2$ \\
Mathur et al. [18] & 0 & $1.41 \pm 0.12$ \\
SCALAR [14] & 0 & $\sim 1.7$ \\
\hline
\end{tabular}

Table 2: Lightest strange-light $0^{+}$meson from lattice QCD.

still not clear. The $0^{++}$mesons potentially contain glueball, tetraquark, meson molecule or even quark-antiquark degrees of freedom.

There are a number of reasons that lattice calculations of the light scalar mesons are challenging. The lattice QCD correlators for scalar mesons are more noisy than for $\rho$ and $\pi$ mesons. The light scalar mesons decay via S-wave decays, so the state decays when its mass equals the sum of the masses of the decay products. This makes it easier to see the effect of the strong decay in lattice calculations, than for states that decay via $\mathrm{P}$-wave, such as $\rho, \Delta$, or Roper resonance.

\subsection{The flavour non-singlet $0^{++}$and $0^{+}$mesons.}

In table 11 collect some pertinent experimental properties of the light flavour non-singlet scalar mesons. For more detailed information see the reviews [12, 13]. The existence of the $\kappa$ meson is controversial (see [14] for a discussion), I use the masses and widths from [15].

Although I am going to loop through the lattice results for the different mesons in table 1 i one by one, it is also important to classify the states into SU3 multiplets or a classification based on tetraquarks. The key questions we want to answer from lattice QCD are: do we see $a_{0}(980)$ with $\bar{q} q$ operators (where $q$ is a generic light quark) and do we see the $\kappa$ meson at all?

In table 2 I collect results for the mass of the lightest $0^{+} \bar{q} s$ meson from lattice QCD calculations. The lattice results in table 2 are consistent with experimental mass of the $K_{0}^{\star}(1430)$, but mostly miss the controversial $\kappa$ particle. All the lattice calculations used $\bar{s} q$ interpolating operators, so may have missed the $\kappa$ state, if it is mostly a tetraquark state, with no overlap with $\bar{s} q$ interpolating operators.

I now discuss the mass of the lightest flavour non-singlet $0^{++}$meson from quenched and unquenched QCD. In quenched QCD there is a ghost contribution [19], due to the $\eta \pi$ contribution, to the scalar correlator that needs to be subtracted off the lattice data. This contribution is included via chiral perturbation theory [19]. I collect together some recent results for the mass of the light $0^{++}$meson from lattice QCD in table 3. I only include quenched data where the $\eta \pi$ contribution has been corrected, hence there is no data before the paper by Bardeen at al. [19] in 2001. 


\begin{tabular}{|c|c|c|}
\hline Group & $n_{f}$ & $m_{a_{0}} \mathrm{GeV}$ \\
\hline Bardeen at al. [19] & 0 & $1.34(9)$ \\
Burch et al. [20] & 0 & $\sim 1.45$ \\
Hart et al. [21] & $2 \mathrm{P}$ & $1.0(2)$ \\
Prelovsek et al. [16] & 2 & $1.58(34)$ \\
Prelovsek et al. [16] & $2 \mathrm{P}$ & $1.51(19)$ \\
Mathur et al. [18] & 0 & $1.42(13)$ \\
\hline
\end{tabular}

Table 3: A collection of results from lattice QCD for the mass of the lightest non-singlet $0^{++}$meson. The P stands for partially quenched.

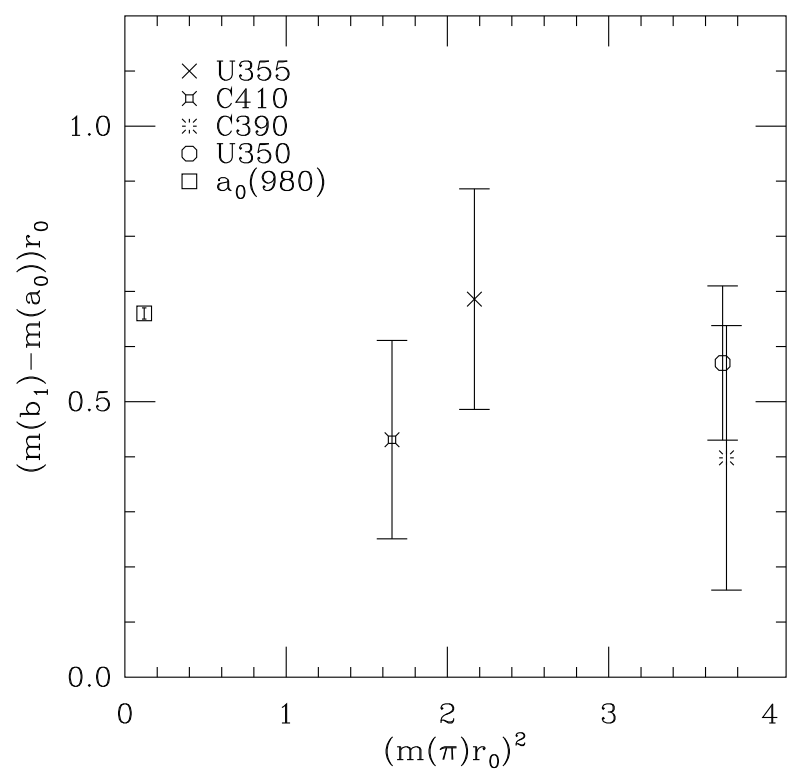

Figure 2: Difference in mass between masses of light $b_{1}$ and $a_{0}$.

McNeile and Michael [17], in an unquenched lattice QCD calculation focused on the mass difference (in the hope that systematics cancel), between the $1^{+-}$and the $0^{++}$mesons. The lattice calculation used gauge configurations from UKQCD's non-perturbatively improved clover action [8], and configurations from CP-PACS's tadpole improved clover program [22]. The results for the mass difference are plotted in figure 2 . Figure 2 shows that the mass of the $1^{+-}$was higher than the $0^{++}$. The final result was $m_{b_{1}}-m_{a_{0}}=221(40) \mathrm{MeV}$, compared to the experimental result of $245 \mathrm{MeV}$. At this conference Lang et al. reported masses for the lightest flavour non-singlet $0^{++}$ consistent with the mass of the $a_{0}(980)$ meson, from an unquenched lattice QCD calculation using chirally improved fermions [23]. 
The previous lattice QCD calculations were in a regime where the quark masses were large enough that the decay $a_{0} \rightarrow \eta \pi$ was forbidden. Now I discuss the new lattice QCD calculations where the decay $a_{0} \rightarrow \eta \pi$ is energetically allowed.

The MILC collaboration [24] originally claimed that they had evidence for $a_{0}$ decay to $\pi \eta$ from their 2+1 calculations with improved staggered fermions. Other decays are discussed in [25]. Later work by the MILC [25] and UKQCD [26] collaborations showed that the lightest state in the flavour non-singlet $0^{++}$channel was actually below the $\pi \eta$ threshold, with improved staggered fermions. This was puzzling, because experimentally the $a_{0} \rightarrow \pi \pi$ decay is forbidden by G parity.

In [27], Prelovsek explained the behaviour of the flavour non-singlet $0^{++}$correlator with improved staggered fermions using staggered chiral perturbation theory. Bernard, DeTar, Fu, and Prelovsek [28] extended the original analysis by Prelovsek, and also applied it to the flavour singlet $f_{0}$ meson. This is a successful theoretical test of the rooting of the staggered determinant, but a larger study, with more sea quark masses, is required to say something specific about the mass of the $a_{0}$ meson.

The ETM collaboration have preliminary results for the mass of the light $0^{++}$meson from a $n_{f}=2$ unquenched lattice QCD calculation with twisted mass fermions. In figure 3 I plot the mass of the light $0^{++}$meson and the $\pi+\eta_{2}$ decay threshold as a function of the square of the pion mass. The mass of the $\eta_{2}$ was computed by Michael and Urbach [4]. Figure 3 shows some evidence for the mass of the $0^{++}$tracking the $\pi+\eta_{2}$ threshold, or at least for it being an open decay channel. The mass of $0^{++}$state is relatively independent of mass and consistent with the mass of $a_{0}(1450)$ meson. As noted by Mathur et al. [18], the mass independence of $0^{++}$is consistent with closeness of the experimental masses of the $a_{0}(1450)$ and $K_{0}(1430)$, if they are both part of the same SU3 octet.

Although the preliminary results from the ETM collaboration are inconsistent with the results by Michael and McNeile [17], some caution is required. We are only just starting to deal with mesons with open decay channels in unquenched lattice QCD calculations. As discussed in section 2, there is a big difference between the lightest flavour singlet pseudoscalar meson in lattice QCD calculations with $n_{f}=2$ and $n_{f}=2+1$ sea quark flavours.

To learn how to deal with mesons with open decays on the lattice we need some simple test cases to validate the lattice methods. The $b_{1}(1235)$ meson is good example, because most people think that it is $\bar{q} q$ state and its width is not too large at $142 \mathrm{MeV}$ [29]. A bad example to study is the $a_{1}(1260)$ with an experimental width of 250 to $600 \mathrm{MeV}$ [29]. The $b_{1}$ meson has the dominant decay $\omega \pi$, but I will plot the $\rho \pi$ decay threshold because the difference between the $\rho$ and $\omega$ correlators are disconnected and are thought to be small.

In figure $4 \mathrm{I}$ plot some preliminary results from the ETM collaboration for the mass of the $b_{1}$ meson with the estimate of the $\omega \pi$ threshold, as a function of the square of the pion mass. The mass of the lightest state in the $b_{1}$ channel does seem to track the decay threshold quite well. I also include results from the MILC collaboration [24].

\subsection{Determining the structure of a meson}

Some questions such "is this state a molecule?" can be reformulated as: "what is the structure of this hadron?". Most lattice QCD calculations just introduce a particular class of interpolating operator, such as $\bar{q} q$ or $\bar{q} \bar{q} q q$ and then check to see what the lowest masses are. It would be useful 


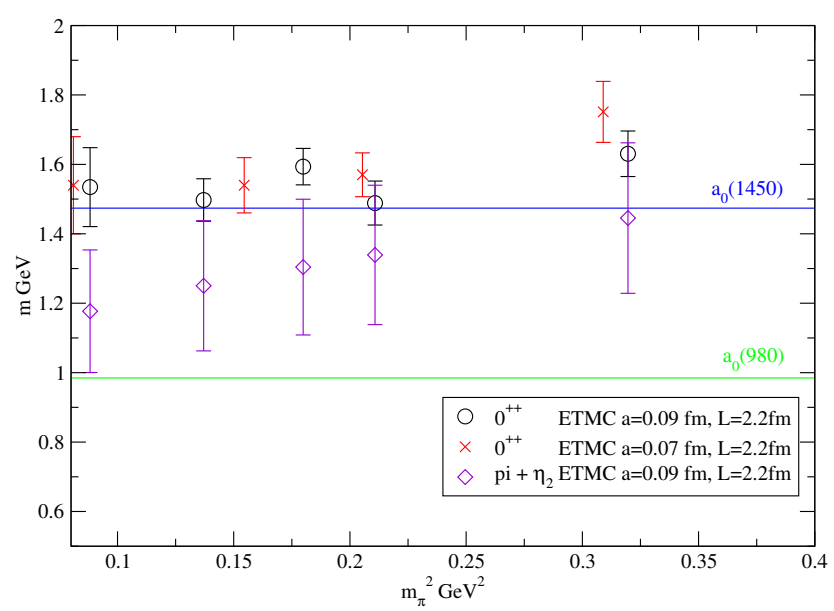

Figure 3: Mass of lightest state in $0^{++}$channel with $\pi \eta_{2}$ decay threshold.

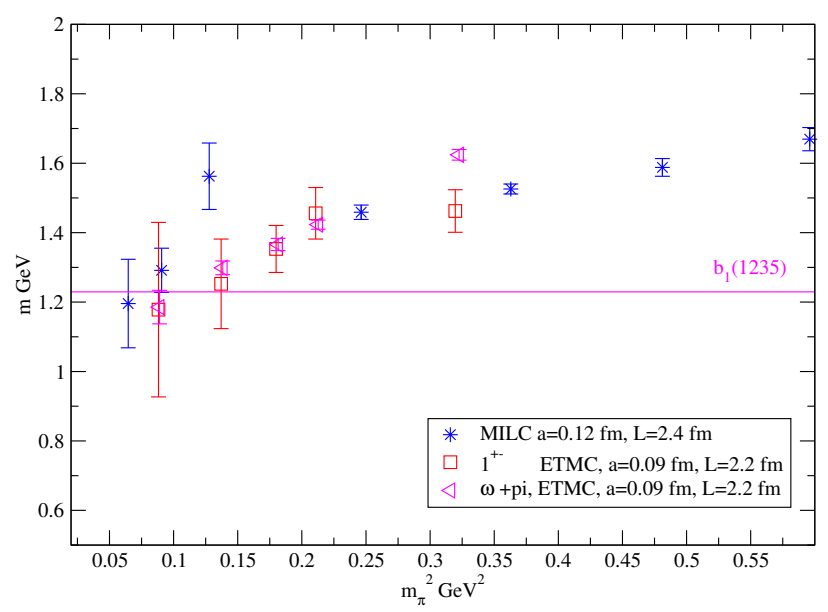

Figure 4: Mass of $b_{1}$ state as a function of square of pion mass

if lattice QCD calculations also tried to look at the structure of mesons. This is after all how continuum phenomenology is done or attempted.

In the past there have been attempts to measure wave functions of hadrons of the form $\bar{\psi}(x+$ $r) \psi(x)$. This can be done by fixing the gauge, or inserting gauge links between the quark and antiquark fields. Because the $\bar{\psi}(x+r) \psi(x)$ operator is restricted to one particular Fock space component these objects are not directly accessible to experiment via a form factor measurement, but are still a valuable theoretical tool. The CLQCD collaboration [30] have recently argued that because they found a node in the lattice wave-function of the first excited $1^{++}$state (a candidate for the $\mathrm{X}(3872)$ ) in charmonium, then the state was a conventional $\bar{c} c$ meson.

The one test that is commonly used in lattice QCD calculations, that include tetraquark degrees of freedom, is to study the volume dependence of the amplitudes. A strong volume dependence indicates that the state is a scattering state and no resonance is formed [31]. 


\begin{tabular}{|c|c|c|}
\hline Group & Method & $f_{a_{0}} \mathrm{MeV}$ \\
\hline Maltman [38] & sum rule & 298 \\
Shakin and Wang [39] & model & 433 \\
Narison [34] & sum rule & $320-390$ \\
\hline
\end{tabular}

Table 4: Some results from models for $f_{a_{0}}$ decay constant.

There are also observables that are sensitive to the structure of the hadron that are also in principle accessible to experiment. For example, leptonic decay constants, strong decay widths, and two photon decay widths.

Pennington [32] has recently extracted the two photon decay width of the $\sigma$ from experiment to be $\Gamma(\sigma \rightarrow \gamma \gamma) \sim 4 \mathrm{keV}$. Pennington notes that value of $\Gamma(\sigma \rightarrow \gamma \gamma)$ can depend quite sensitively on the quark content of the $\sigma$ [32]. Thanks to Dudek and Edwards [33] we now have a formalism to compute two photon widths on the lattice. Dudek and Edwards [33] compute $\Gamma\left(\chi_{0} \rightarrow \gamma \gamma\right)=$ $2.4 \pm 1.0 \mathrm{keV}$, from a quenched QCD calculation. It would be interesting to do a similar calculation for light scalars.

Narison [34] proposed to use the leptonic decay constant of the non-singlet $0^{++}$mesons to determine the structure of the $a_{0}$ meson. The $f_{a_{0}}$ decay constant of the light flavour non-singlet $0^{++}$meson has been computed using unquenched lattice QCD [17].

$$
\left\langle 0|\bar{q} q| a_{0}\right\rangle=M_{a_{0}} f_{a_{0}}
$$

See [17] for a further discussion of this decay constant and the connection with the electroweak current. The decay constant $f_{a_{0}}$ depends on the renormalisation scale, however here I just do a qualitative comparison. The experimental measurement of the decay constant in equation 3.1 via $\tau$ decay is discussed by Diehl and Hiller [35].

A molecule of two mesons should have a very small "wave-function" at the origin, hence $f_{a_{0}}$ should be small. The definition of $f_{a_{0}}$ is similar to that of the pion decay constant. Hence we mean "small" relative to $130 \mathrm{MeV}$. The other measured decay constants of pseudoscalar mesons are within a factor of 2.5 to the pion decay constant [29]. The only exception is the decay constant of the $\pi(1300)$ that is suppressed [36, 37]. A large value for decay constant $f_{a_{0}}$ does not rule out a $\overline{q q} q q$ multi-quark meson.

Using gauge configurations from UKQCD and CP-PACS, McNeile and Michael computed $f_{a_{0}} \sim 480 \mathrm{MeV}$. In table $\emptyset \mathrm{I}$ collect some results for the value of $f_{a_{0}}$ from model calculations and sum rules (see also [40]). A lattice QCD calculation of the decay constant of the scalar heavy-light mesons was reported in [41].

Computing the decay width of a hadron is also very a valuable way of identifying a state on the lattice. In [17], it was reported that the experimental hadron coupling for the decays $a_{0}(980) \rightarrow K \bar{K}$ and $a_{0}(1450) \rightarrow K \bar{K}$ were 0.9 and 0.5 respectively. A lattice calculation [17] found that the lightest hadron in the $0^{++}$correlator had a coupling to $K \bar{K}$ of $\approx 1$, thus providing additional evidence that the lightest state was the $a_{0}(980)$.

\subsection{Flavour singlet $0^{++}$mesons}

In pure SU3 gauge theory, Morningstar and Peardon [42] found 13 glueballs with masses 


\begin{tabular}{|c|c|c|c|c|}
\hline Meson & $\mathrm{M} \mathrm{MeV}$ & $\Gamma \mathrm{MeV}$ & decay & comment \\
\hline$f_{0}(600)(\sigma)$ & 441 & 544 & $\pi \pi$ & tetraquark, molecule, $\bar{q} q$ \\
$f_{0}(980)$ & 980 & $40-100$ & $\pi \pi$ & tetraquark, molecule, $\bar{q} q$ \\
$f_{0}(1370)$ & $1200-1500$ & $200-500$ & $\pi \pi$ & $\bar{q} q$, glueball \\
$f_{0}(1500)$ & 1505 & 109 & $\pi \pi$ & $\bar{q} q$, glueball \\
$f_{0}(1710)$ & 1724 & 137 & $\bar{K} K$ & $\bar{q} q$, glueball \\
\hline
\end{tabular}

Table 5: Light flavour singlet $0^{++}$mesons from the PDG. The dominant strong interaction decay is reported.

under $4 \mathrm{GeV}$. This raises the question as to whether there is any evidence for glueballs in nature, or are glueballs just theoretical constructs that are only of interest to test ADS/CFT techniques for example. One way we can answer this question on the lattice is to study the effect of sea quark dynamics on the glueball masses. I will focus on the $0^{++}$state, as this is where the most work has been done in unquenched lattice QCD, and where there are possible experimental candidates. Hart and Teper [43] only found a signal for the $0^{++}$and $2^{++}$states from unquenched calculations.

I summarise some pertinent experiment results for the flavour singlet $0^{++}$mesons in table 5 . The $f_{0}(600)(\sigma)$ is particularly interesting. It was proposed in 1955 , but only entered the PDG summary tables in 2002 [18]. The parameters for the $f_{0}(600)$ comes from the work of Caprini et al. [44], while the PDG summary quotes a mass in the range 400 to $1200 \mathrm{MeV}$ [29].

Morningstar and Peardon [42] obtained $M_{0^{++}}=1730(50)(80) \mathrm{MeV}$ for the mass of the lightest $0^{++}$glueball from quenched QCD. Chen et al. [45] recently found $M_{0^{++}}=1710(50)(80) \mathrm{MeV}$. The quark model predicts that there should only be two $0^{++}$mesons between 1300 and $1800 \mathrm{MeV}$, so if the mixing between the glueball and $\bar{q} q$ operators is weak, then the $0^{++}$glueball is hidden inside the $f_{0}(1370), f_{0}(1500)$ and $f_{0}(1710)$ mesons.

The old work by Weingarten and Lee [46] on glueball- $\bar{q} q$ mixing introduced a mixing matrix between the glue and $\bar{q} q$ states. The matrix elements were estimated in quenched QCD. Weingarten and Lee [46] predicted that the $f_{0}(1710)$ meson was $74(10) \% 0^{++}$glueball, and hence the mixing between the $0^{++}$glueball and $\bar{q} q$ states was weak. Weingarten and Lee's [46] calculations were critiqued in [47]. Also their results were probably effected by the quenched artifact in the nonsinglet $0^{++}$correlator [19]. As discussed in section \&, now that $\eta, \eta^{\prime}$ mixing is now being studied in $2+1$ lattice QCD calculations, then the mixing between light and strange flavour singlet scalar operators can in principle also be included.

There are claims that the continuum phenomenology is more consistent with the strong mixing between $0^{++}$glueball and $\bar{q} q$ states. In this case there could be a sizable contributions from glueball interpolating operators to the $f_{0}(600)$ or $f_{0}(980)$ mesons.

The SESAM collaboration studied the glueball spectrum on unquenched lattices [48]. McNeile and Michael studied the light $0^{++}$spectrum with unquenched QCD [47] at a coarse lattice spacing and found the mass of the lightest flavour singlet $0^{++}$meson was very light. Using $0^{++}$ glueball operators, Hart and Teper [43] found that

$$
M_{0^{++} \text {UNquenched }}=0.85(3) M_{0^{++}} \text {Quenched }
$$

at a fixed lattice spacing of $0.1 \mathrm{fm}$. 


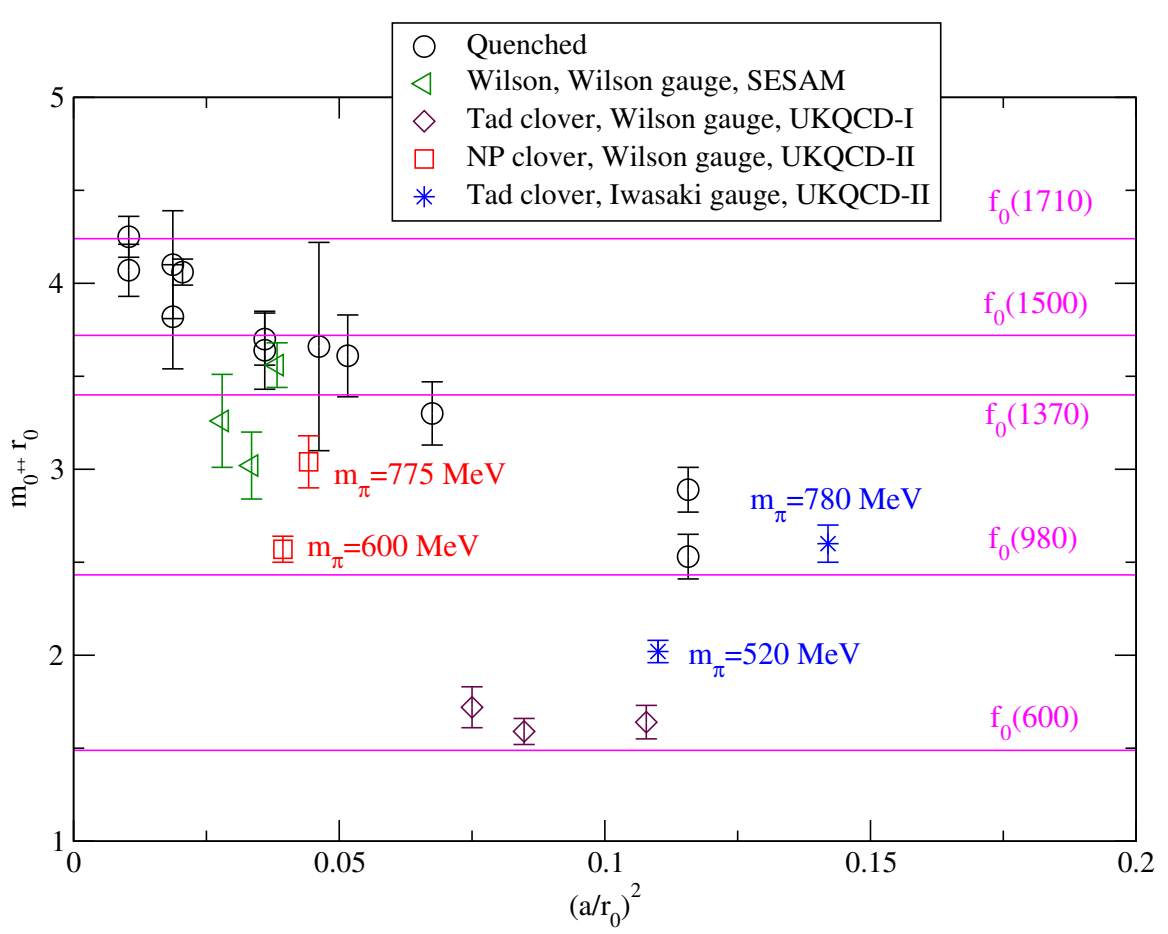

Figure 5: Summary of unquenched results for lightest flavour singlet $0^{++}$mesons from [50]. The unquenched results are from SESAM [48], UKQCD-I [47], and UKQCD-II [50].

Unfortunately, the existing unquenched lattice QCD calculations of the flavour singlet $0^{++}$ mesons don't have the range of lattice spacings where a continuum extrapolation can be attempted. In quenched QCD it was found that the lattice spacing dependence of the mass of the $0^{++}$glueball was strong. The use of a Symanzik improved gauge action by Chen et al. [45] and, Morningstar and Peardon [42], produced a slightly smaller slope with lattice spacing of the scalar $0^{++}$glueball mass, than for calculations that used the Wilson plaquette action. This is relevant to unquenched calculations, because any suppression of the mass of the flavour singlet $0^{++}$mass may be due to lattice spacing effects.

The SCALAR collaboration [49], used unquenched lattice QCD, with Wilson fermions and the Wilson gauge action, to study the $0^{++}$mesons. At a single lattice spacing a $\sim 0.2 \mathrm{fm}$, with $\bar{q} q$ interpolating operators only, they obtain $m_{\bar{q} q} \sim m_{\rho}$. At the very least this result needs to be checked with a continuum extrapolation.

In unquenched QCD, both glue and $\bar{q} q$ states will couple to singlet $0^{++}$mesons, so it is better to do a variational fit with both types of operators as basis interpolating operators. The variational technique analysis of the singlet $0^{++}$mesons was done by Hart et al. [50]. A combined fit to $0^{++}$glue and $\bar{q} q$ interpolating operators with two types of spatial smearing sources was done. The calculation used non-perturbative improved clover action at a single lattice spacing [8]. Configurations from CP-PACS [22] with the Iwasaki gauge action and tadpole improved clover action were also used in the analysis. A summary plot of the results is in figure 5 . The data with the bursts and squares (with the pion masses written near them) in figure 5 shows an additional reduction of the 
mass of the $0^{++}$state over the pure glueball operators, as used by Hart and Teper [43]. The data with the Iwasaki action should be less affected by lattice artifacts [22].

Mathur at al. [18] recently claimed to get a result for the $f_{0}(600)(\sigma)$ from quenched lattice QCD with pion masses as low as $180 \mathrm{MeV}$. Using the interpolating operator $\bar{\psi} \gamma_{5} \psi \bar{\psi} \gamma_{5} \psi$ they obtain $m_{f_{0}(600)} \sim 550 \mathrm{MeV}$. This interpolating operator has a disconnected contribution that they computed and found to be small. The key part of this work is a three state fit ( $\pi(p=0) \pi(p=0)$ , $f_{0}(600), \pi\left(p=\frac{2 \pi}{L}\right) \pi\left(p=\frac{-2 \pi}{L}\right)$ using the Bayes adaptive curve fitting algorithm [51]. They studied the finite volume effects to distinguish the signal for the resonance from the $\pi \pi$ scattering states [31].

I have a number of concerns about their result. For example, can quenched QCD really be used to calculate the mass of a meson with a mass of $440 \mathrm{MeV}$ and width of $544 \mathrm{MeV}$ [44]? Mathur et al. [31] computed the mass of the $K^{\star}(1430)$, with a width of $290 \mathrm{MeV}$ to be $1.41 \pm 0.12$.

Another concern is the use of the Bayes adaptive fitting [51] algorithm. This is a clever way of doing a multi-exponential fit to a single correlator. The Bayes adaptive fitting method was used by the Kentucky group to study the Roper resonance [52] in quenched QCD. Other groups have not confirmed this (see Lasscock et al., [53] for a review), partly because the Kentucky group worked with much lighter pions than other groups

Sugamuma et al. [54] used quenched QCD to study light $0^{++}$states with $q q \overline{q q}$ interpolating operators. Hybrid boundary conditions and the Maximum Entropy Method were used to study correlators. No localized resonance of the form $q q \overline{q q}$ was found in the quark mass region of $m_{s}<m_{q}<2 m_{s}$.

\section{Brief overview of status of unquenched QCD}

There have been individual plenary reviews for some of the recent large scale lattice QCD calculations [55, 56]. In this section I compare some results from different lattice QCD calculations. I used $r_{0}=0.469 \mathrm{fm}$ from HPQCD and MILC collaborations [25, 57] to consistently determine the lattice spacing. I will discuss this more in section 5. This will make the comparison of some group's results against experiment slightly worse, but it is necessary to use a common value of $r_{0}$ to compare the results of different groups. I mostly use recently published data, rather than use the very latest results presented at this conference.

I compare results at fixed lattice spacing. The results from different actions only need to agree in the continuum limit. In the past there was a controversy that the quenched Edinburgh plots for the Wilson and staggered actions did not agree in the continuum limit [58]. This was largely resolved by better extrapolations to the continuum limit [59, 60]. All the recent large scale calculations use improved lattice formulations, so the corrections to the continuum limit should be small (but still need to be quantified of course).

\subsection{The pion decay constant}

In figure 6 I plot the pion decay constant in physical units as a function of the square of the pion mass in physical units. I have applied the finite size corrections from Colangelo et al. [61]. The investigation of finite size effects is an active area of study at the moment (see for example [55]). 


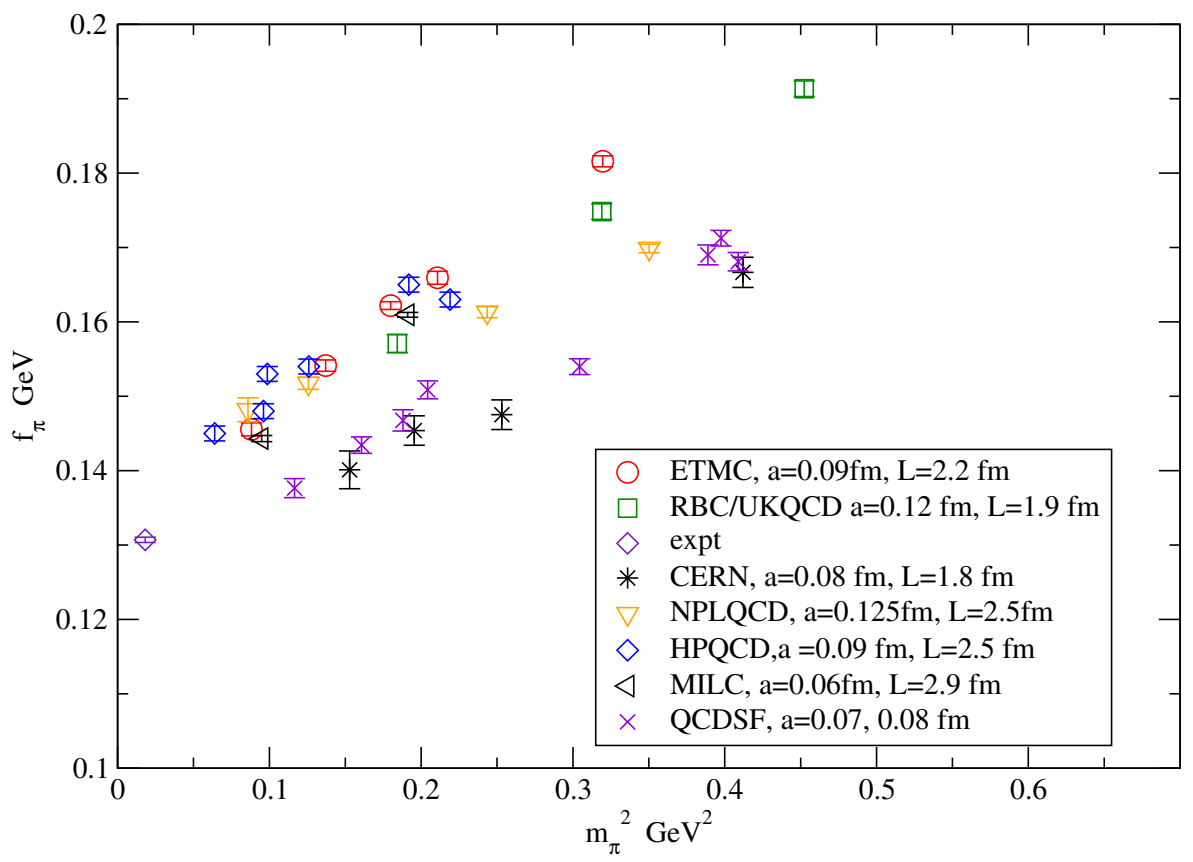

Figure 6: The pion decay constant as a function of the square of the pion mass. There is data from MILC [62], NPLQCD [63], HPQCD [64], CERN [65, 66] and QCDSF [67]

All the data in figure 6 either use definitions of $f_{\pi}$ with an automatic matching factor of 1 , or a renormalisation factor that is obtained via a nonperturbative method.

I find it very encouraging that the results, in figure 6, from MILC [62] (the new results from the superfine run), NPLQCD [63], HPQCD [64] and ETM collaborations [68] cluster very closely together.

The data from the CERN [65, 66] and QCDSF [67] groups slightly disagree with other groups at the $\sim 4 \mathrm{MeV}$ level. There could be several reasons for this. This could be a difference due to $O\left(a^{2}\right)$ terms, or the way $Z_{A}$ is treated. It would be interesting to do a similar comparison for heavy-light decay constants [69].

The CERN group [65, 66] presented their results as a ratio of the decay constant to a reference pion mass. This analysis method was used by the ALPHA collaboration [70, 71] to look for chiral logs, or quenched artifacts, in the pion mass and decay constant. As previously noted by Jansen [59], the ratio method hides a lot of systematic errors. In figure 7 I use the ratio method to compare the data from the CERN group [65, 66] with that from the ETM collaboration [68]. The agreement is much better in the ratio plot, than comparing just the decay constant.

Now that the quality of unquenched lattice QCD calculations is improving, we may need to purge the subject of analysis techniques that were developed when the data was poor. For example, the Edinburgh (or APE) plots of the ratio of the nucleon mass to vector meson mass as a function of the ratio of the pion mass to vector meson mass, mix up the problems of the sensitive chiral extrapolation of the nucleon mass with the difficult problem of the effect of decay of the $\rho$ meson. 


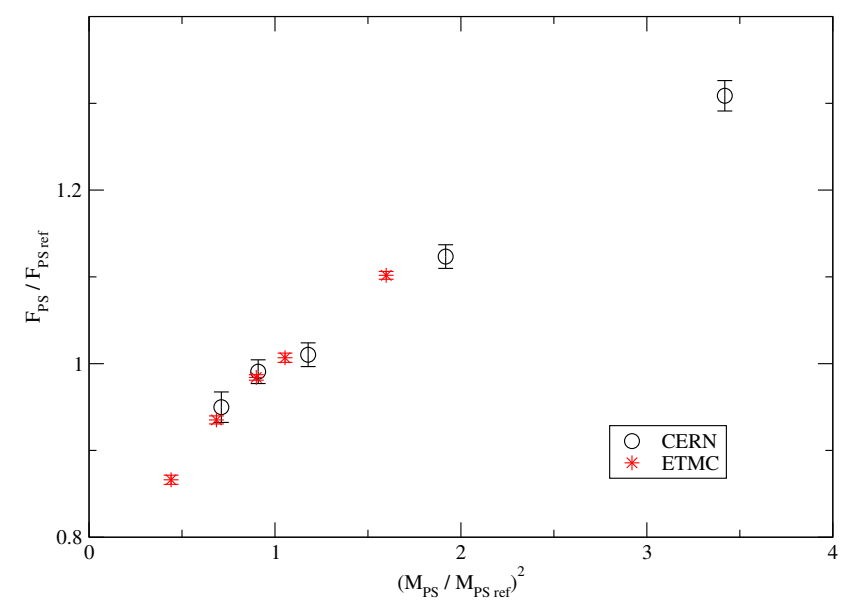

Figure 7: Ratio of decay constants. Comparing the results from ETM collaboration [68] and the CERN group [66].

Usually both the vector and nucleon masses have fairly large errors, but there is no cancellation when the ratio is taken, so the Edinburgh plot tends to amplify the errors.

\subsection{The $\rho$ meson}

In figure $8 \mathrm{I}$ plot the mass of the light vector meson as a function of the square of the pion mass, from lattice QCD calculations that use improved staggered, domain wall, and twisted mass fermions. The data in figure 8 are remarkably consistent, although the statistical errors need to be reduced on some results.

In the real world the $\rho$ decays into two pions, via a P-wave decay. The threshold for decay is $2 \sqrt{m_{\pi}^{2}+\left(\frac{2 \pi}{L}\right)^{2}}$ where $L$ is the side of the box. The CERN group [65, 66] found excited masses for the $\rho$ channel that were consistent with $2 \sqrt{m_{\pi}^{2}+\left(\frac{2 \pi}{L}\right)^{2}}$. It is more kinematically favourable to study the decay of the $\rho$ meson with one unit of momentum to decay to a pion at rest and a pion with one unit of momentum [73, 74].

Michael and Urbach for the ETM collaboration [Џ], estimated the mixing element between $\rho$ and $\pi \pi$ from a three point function. This mixing produced a 5\% shift in the mass of the lightest $\rho$ using the method in [73]. This suggests that the mass of the $\rho$ meson in figure 8 from the ETMC collaboration is largely unaffected by the two $\pi$ decay.

The $\rho$ decay will effect the chiral extrapolation model used to extrapolate the mass of the rho meson. The Adelaide group have studied different regulators [75, 76] for the effective field theory of $\rho$ decay. This produced additional mass dependence for very light pion masses. These type of chiral extrapolation models for the $\rho$ meson were not widely used to analyse lattice data. One reason for this was that the fit models showed behaviour outside simple linear or quadratic mass dependence in regimes where there were no lattice data. Now that many groups have unquenched data with pion masses in the 300 to $400 \mathrm{MeV}$ regime, now is the time to revisit at this issue. Bruns and Meißner [77] have published a new chiral extrapolation formulae for the mass of the $\rho$ meson. 


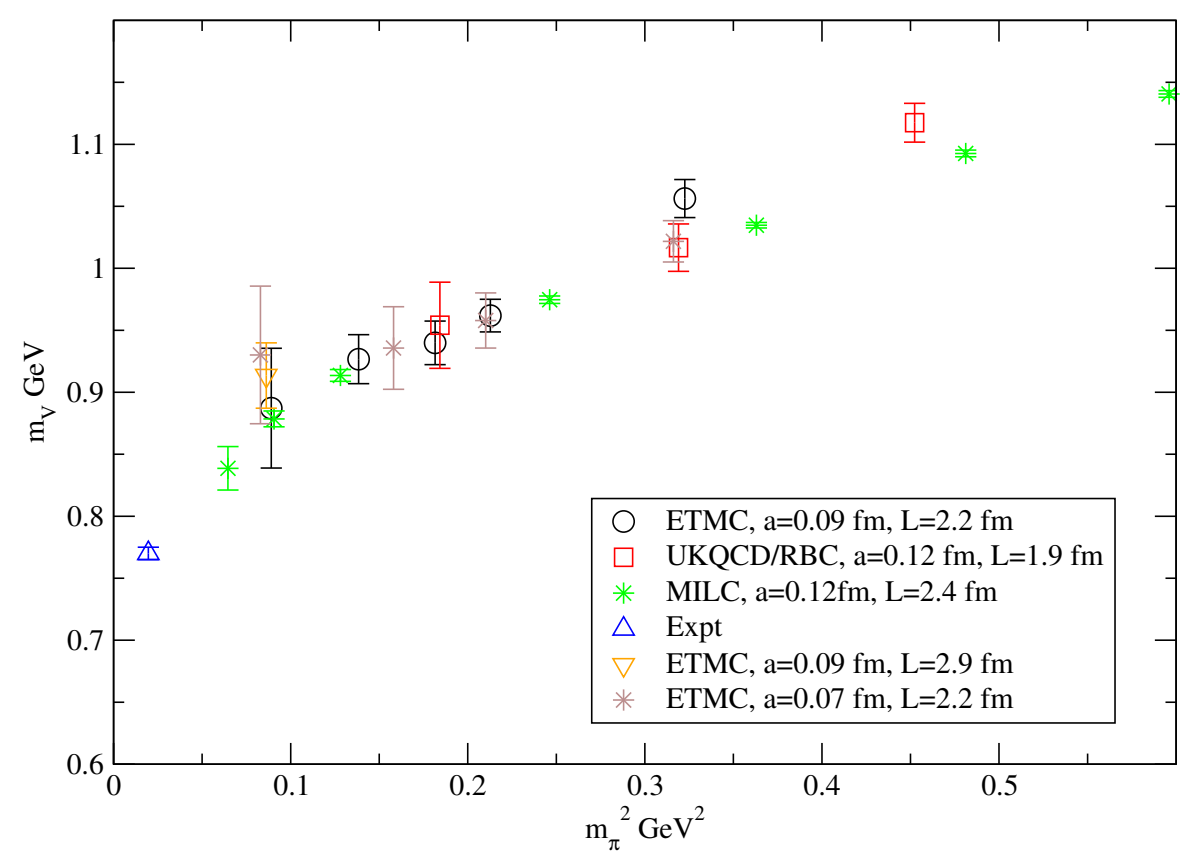

Figure 8: The mass of the light vector meson as a function of the square of the pion mass. I include preliminary data from ETMC [68], and published data from RBC-UKQCD [72] and the MILC collaboration [24]

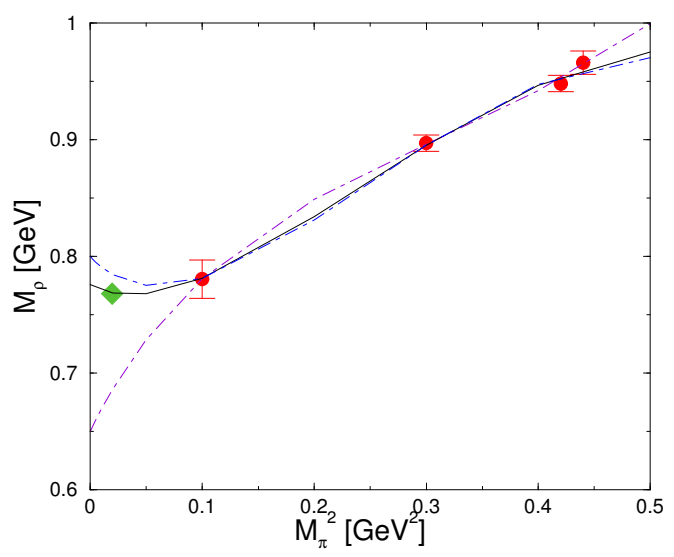

Figure 9: Quark mass dependence of the mass of the $\rho$ [77].

The derivation used a modified $\overline{M S}$ regulator and a power counting scheme.

$$
M_{\rho}=M_{\rho}^{0}+c_{1} M_{\pi}^{2}+c_{2} M_{\pi}^{3}+c_{3} M_{\pi}^{4} \ln \left(\frac{M_{\pi}^{2}}{M_{\rho}^{2}}\right)
$$

The term with the $c_{3}$ coefficient is due to the self energy (in the infinite volume limit). Bruns and Meißner [77] recommend that the size of the $c_{i}$ coefficients obtained from the fits be checked against other estimates (eg. from $\omega \rho \pi$ coupling). Figure 9 from the paper by Bruns and Meißner [77] 
shows the effect of the extrapolation model in equation 4.1 on older CP-PACS data. See [78] for an another analysis of the $\rho$ decay on the mass of $\rho$ meson.

\section{Determining the lattice spacing}

To convert the results from unquenched lattice calculations from lattice units into physical units, requires that the lattice spacing is determined. In principle this requires that a single physical number be sacrificed to determine the lattice spacing. There are problems with many of the obvious choices of quantities to use. For example the mass of the nucleon is very sensitive to the chiral extrapolations of the mass to physical quark masses. Now that most groups are using some kind of non-perturbative renormalisation in the calculation of the $f_{\pi}$ this is in principle a good quantity to use to determine the lattice spacing. Unfortunately, the determination of $f_{\pi}$ also requires a careful chiral extrapolation. Also as Marciano [79] pointed out the ratio $\frac{f_{K}}{f_{\pi}}$ can be used to extract the CKM matrix element $V_{u s}$.

Quantities that are not good choices to determine the lattice spacing are hadrons that decay via the strong interaction [80]. Given the discussion about quark mass dependence of the mass of the light vector meson in section 4.2, it is clear that using the $\rho$ mass or $K^{\star}$ mass to set the scale is not a good idea, because we don't have full theoretical control of the chiral extrapolation. The problem of using the $\rho$ mass to set the scale has been known for a long time, for example it was discussed by DeGrand at the lattice 90 conference [81], although it is still being used [72].

However, I don't think we should give up studying the properties $\rho$ or $K^{\star}$ mesons using lattice QCD. Obtaining an accurate value of the $\rho$ mass from lattice QCD is prerequisite to calculations of hadrons such as the Roper resonance or scalar mesons. There are also a number of important semi-leptonic weak decays, such as $B \rightarrow \rho+\gamma, B \rightarrow K^{\star}+\gamma$ that involve a $\rho$ meson in the final state.

One popular way to determine the lattice spacing is to use a quantity called $r_{0}$ [82], determined from the heavy quark potential, via

$$
F\left(r_{0}\right) r_{0}^{2}=1.65
$$

where $F$ is the force of the heavy quark potential. The value of $r_{0}$ in lattice units can be obtained very accurately from lattice calculations. When Sommer [32] introduced $r_{0}$ as he assigned $r_{0}=$ $0.49 \mathrm{fm}$, from continuum heavy quark potentials, with an estimated uncertainty of $10 \%$. The value of $r_{0}$ has largely replaced the use of the string tension to determine the lattice spacing.

The MILC/HPQCD collaborations have determined $r_{0}=0.469(7) \mathrm{fm}$ from the $2 \mathrm{~S}-1 \mathrm{~S}$ mass splitting in $\Upsilon$ from unquenched lattice QCD calculations with 2+1 flavours of improved staggered quarks [57]. A consistent lattice spacing is determined from other quantities, such as other splittings in the $\Upsilon$ system, $f_{\pi}$, and mass splittings in charmonium [80]. This value of $r_{0}$ is crucial to the phenomenology produced by the improved staggered program. For example, if I naively change from using $r_{0}=0.469 \mathrm{fm}$ to $r_{0}=0.49 \mathrm{fm}$, with HPQCD's recent result for $f_{D_{s}}=241$ (3) MeV, then their "new" number is 231(3) MeV [64]. This is a shift of $3 \sigma$. As the HPQCD collaboration push to ever higher precision the current error on $r_{0}$ needs to be reduced [64].

Other collaborations, for example RBC-UKQCD [72], use $r_{0}=0.49 \mathrm{fm}$ to set the lattice spacing, based on consistency with $\rho$ mass and the $K^{\star} / K$ mass ratio. As reviewed by Boyle [56], the 


\begin{tabular}{|c|c|c|c|}
\hline Group & $n_{f}$ & Method & $r_{0} \mathrm{fm}$ \\
\hline Sommer [82] & - & quark model & $0.49_{-5}^{+0}$ \\
Morningstar and Peardon [83] & 0 & quenched summary & $0.48(2)$ \\
\hline UKQCD [8] & 2 & $\mathrm{~K} / \mathrm{K}^{*}$ & 0.55 \\
JLQCD [84] & 2 & $m_{\rho}$ & $0.497(-9)(13)$ \\
JLQCD [84] & 0 & $m_{\rho}$ & $0.5702(75)(50)$ \\
QCDSF [85] & 2 & summary nucleon masses & $0.47(3)$ \\
\hline QCDSF [78] & 2 & $\underline{f_{\pi}}$ & $0.45(1)$ \\
ETMC [68] & 2 & $f_{\pi}$ & $0.454(7)$ \\
HPQCD/MILC/FNAL [80, [7] & $2+1$ & Upsilon \& ratio plot & $0.469(7)$ \\
\hline
\end{tabular}

Table 6: Summary of lattice determinations of $r_{0}$.

RBC-UKQCD collaboration are now obtaining lower values of $r_{0}$ than $0.49 \mathrm{fm}$, when they set the lattice spacing using hadrons that are stable under the strong interactions, such as the $\Omega$.

The value of $r_{0}$ can be determined from lattice calculations. In table 6I collect some estimates of the value of $r_{0}$ from lattice QCD calculations. From the summary of the results in table 6 we see that the recent unquenched calculations are starting to report values of $r_{0}$ between 0.44 to $0.48 \mathrm{fm}$.

I am being slightly hypocritical here, because for clover action with $m_{q}>m_{s} / 2$ we used to argue that by using $r_{0} \sim 0.49 \mathrm{fm}$ some systematics might cancel [41].

One problem with using $r_{0}$ to set the scale is that $r_{0}$ needs a chiral extrapolation to this massless limit. See Sommer et al. [86], Aoki [58] and Bhattacharya et al. [87] for discussions about the mass dependence of $r_{0}$. Some part of the mass dependence of $r_{0}$ may be physical, and some part may be a lattice artifact. In figure 10I plot $r_{0}$, with the intercept fixed at $0.467 \mathrm{fm}$, for a number of different lattice formalisms, against the square of the pion mass in physical units.

The clover action is only an onshell improved action. In the ALPHA formalism for the improvement of the clover action, the leading lattice artifact part of the linear mass dependence of $r_{0}$ is related to the $b_{g}$ improvement coefficient.

$$
\hat{g}_{0}^{2}=\left(1+b_{g} a m_{q}\right) g_{0}^{2}
$$

Fully $O(a)$ improved actions, such as domain wall or twisted mass fermions should have no $\mathrm{O}\left(\mathrm{a} m_{q}\right)$ lattice artifacts, and reduced dependence on the quark mass on $r_{0}$. I note that RBCUKQCD [88, 72] linearly extrapolate $r_{0}$ with quark mass, but the ETM collaboration [68] extrapolate $r_{0}$ quadratically in quark mass to the chiral limit.

My naive understanding of the phrase "decoupling of continuum and chiral limits" about fermion operators that are approximate solutions to the Ginsparg-Wilson relation suggested that mass dependence of $r_{0}$ for overlap/DWF actions should be small. The data in figure 10 doesn't appear to show reduced mass dependence for the domain wall data. The domain wall data is at a coarser lattice spacing than for the other actions, so I may be making an unfair comparison.

The MILC collaboration [24] have chosen to work at fixed lattice spacing rather than fixed $\beta$, so I have not included their results in figure 10. As the quark mass is changed, $\beta$ is changed to keep $r_{0} / a$ constant. This was also the strategy of the improved clover calculations performed by the UKQCD collaboration [8, 90]. 


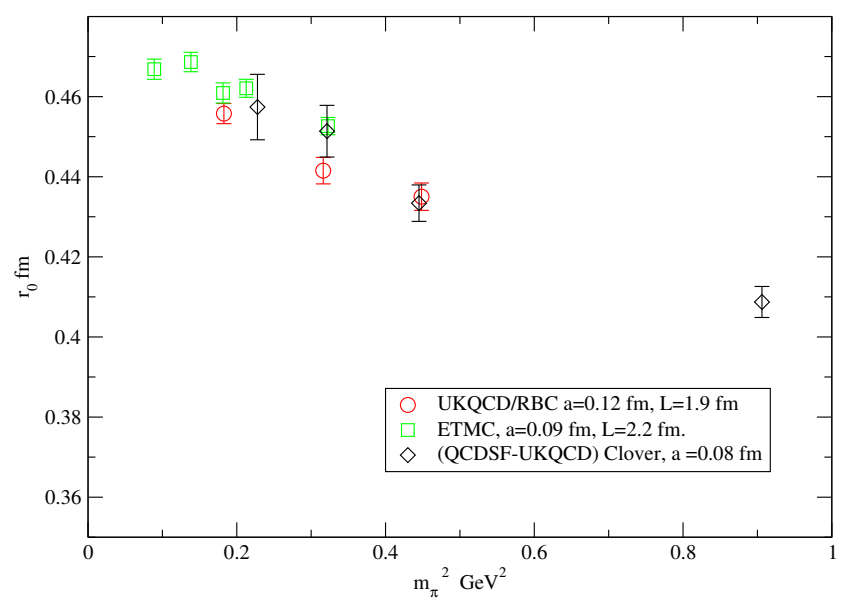

Figure 10: The value of $r_{0}$ as a function of the square of the pion mass, using data from RBC-UKQCD [72], QCDSF-UKQCD [89], and ETMC [68]

In this section I am not advocating that $r_{0}$ should always be used to set the lattice spacing in every calculation. However, I do hope that eventually there will be consensus between different lattice QCD calculations on a final value for $r_{0}$ in physical units. It is certainly a crucial cross-check on the results from the improved staggered program.

\section{Conclusions}

There is still no consensus as to whether $\bar{q} q$ operators in lattice QCD calculations are coupling to the $a_{0}(980)$ meson. To clear up the many questions about the spectrum of the $0^{++}$scalar mesons, unquenched lattice QCD calculations with tetraquark interpolating operators are required. There is "some" evidence that the flavour singlet $0^{++}$interpolating operators, in unquenched lattice QCD calculations, are coupling to states around or below a $1 \mathrm{GeV}[50]$. Although a continuum extrapolation is required for definite results and the open decay channel issue needs to be studied.

After all the successful work on algorithms for reducing the mass of the sea quarks in lattice QCD calculations, we are now starting to study light mesons with open S-wave decay channels. I presented some evidence from the ETM and MILC collaborations, that the $b_{1}$ meson has an open strong decay channel. This type of issue will be of increasing importance for lattice studies of particles that decay via the strong force. For example, the MILC collaboration [91] claimed to see problems with the light exotic $1^{-+}$meson, because of an open decay channel. Eventually, the issue of dealing with resonances in lattice QCD will be dealt with by Lüscher's technique [92]. Until then pragmatic approaches to studying strong decays on the lattice are still important. This year Lüscher's technique for resonances was applied to the $\rho$ meson for the first time, by the CP-PACS collaboration [74].

In the past few years the MILC collaboration [24, 25] have been doing the unquenched calculations with the lightest pions, largest physical volumes, biggest range of lattice spacing. This year mostly due to algorithm improvements, and partly due to bigger machines other collaborations now 
have lattice QCD results that are comparable to the quality of those from the MILC collaboration. It is important to do cross-checks on the results from different lattice QCD formalisms, as part of the quest for errors at the percent level. Many of the older ways to compare results from different lattice QCD calculations may no longer be appropriate to this high precision era of lattice QCD.

\section{Acknowledgements}

I thank Christine Davies and Chris Michael for reading the paper. I thank the ETM collaboration for allowing me to use preliminary results for the $b_{1}$ and $a_{0}$ masses. I thank Chris Michael, Gerrit Schierholz, Roger Horsley, Claude Bernard, Luigi Del Debbio, for discussions and for sending me data.

\section{References}

[1] S. Basak et. al., Lattice qcd determination of patterns of excited baryon states, arXiv: 0709.0008 [hep-lat].

[2] E. S. Swanson, The new heavy mesons: A status report, Phys. Rept. 429 (2006) 243-305, [hep-ph/0601110].

[3] C. McNeile, Lattice approach to threshold states, hep-ph/0610139.

[4] ETM Collaboration, . C. Michael and C. Urbach, Neutral mesons and disconnected diagrams in twisted mass qcd, arXiv:0709.4564 [hep-lat].

[5] E. B. Gregory, A. C. Irving, C. M. Richards, and C. McNeile, Methods for pseudoscalar flavour-singlet mesons with staggered fermions, arXiv:0709.4224 [hep-lat].

[6] CP-PACS Collaboration, V. I. Lesk et. al., Flavor singlet meson mass in the continuum limit in twoflavor lattice qcd, Phys. Rev. D67 (2003) 074503, hep-lat/0211040.

[7] UKQCD Collaboration, C. R. Allton et. al., Improved wilson qcd simulations with light quark masses, Phys. Rev. D70 (2004) 014501, hep-lat/0403007].

[8] UKQCD Collaboration, C. R. Allton et. al., Effects of non-perturbatively improved dynamical fermions in qcd at fixed lattice spacing, Phys. Rev. D65 (2002) 054502, [hep-lat/0107021].

[9] T. Feldmann, Quark structure of pseudoscalar mesons, Int. J. Mod. Phys. A15 (2000) 159-207, [hep-ph/990749].

[10] JLQCD Collaboration, S. Aoki et. al., An estimate of the eta and eta' meson masses in $n(f)=2+1$ lattice qcd, PoS LAT2006 (2006) 204, hhep-lat/0610021].

[11] M. Creutz, Why rooting fails, arXiv:0708.1295 [hep-lat].

[12] K.-F. Liu, Pattern of light scalar mesons, arXiv:0706.1262 [hep-ph]

[13] M. R. Pennington, Scalars in the hadron world: The higgs sector of the strong interaction, Int. J. Mod. Phys. A21 (2006) 747-756, [hep-ph/0509265].

[14] H. Wada et. al., Lattice study of low-lying nonet scalar mesons in quenched approximation, hep-lat/0702023.

[15] S. Descotes-Genon and B. Moussallam, The $k 0 *(800)$ scalar resonance from roy-steiner representations of pi k scattering, Eur. Phys. J. C48 (2006) 553, [hep-ph/0607133]. 
[16] S. Prelovsek, C. Dawson, T. Izubuchi, K. Orginos, and A. Soni, Scalar meson in dynamical and partially quenched two-flavor qcd: Lattice results and chiral loops, Phys. Rev. D70 (2004) 094503, [hep-lat/0407037].

[17] UKQCD Collaboration, C. McNeile and C. Michael, Properties of light scalar mesons from lattice qcd, Phys. Rev. D74 (2006) 014508, [hep-lat/0604009.

[18] N. Mathur et. al., Scalar mesons a0(1450) and sigma(600) from lattice qcd, hep-ph/0607110

[19] W. A. Bardeen, A. Duncan, E. Eichten, N. Isgur, and H. Thacker, Chiral loops and ghost states in the quenched scalar propagator, Phys. Rev. D65 (2002) 014509, hep-lat/ 0106008 .

[20] T. Burch et. al., Excited hadrons on the lattice: Mesons, Phys. Rev. D73 (2006) 094505, [hep-lat/0601026].

[21] UKQCD Collaboration, A. Hart, C. McNeile, and C. Michael, Masses of singlet and non-singlet 0++ particles, Nucl. Phys. Proc. Suppl. 119 (2003) 266-268, [hep-lat/0209063].

[22] CP-PACS Collaboration, A. Ali Khan et. al., Light hadron spectroscopy with two flavors of dynamical quarks on the lattice, Phys. Rev. D65 (2002) 054505, hep-lat/0105015].

[23] R. Frigori et. al., Dynamical chirally improved quarks: First results for hadron masses, arXiv:0709.4582 [hep-lat].

[24] C. W. Bernard et. al., The qcd spectrum with three quark flavors, Phys. Rev. D64 (2001) 054506, [hep-lat/0104002].

[25] C. Aubin et. al., Light hadrons with improved staggered quarks: Approaching the continuum limit, Phys. Rev. D70 (2004) 094505, [hep-lat/ 0402030 ].

[26] E. B. Gregory, A. C. Irving, C. C. McNeile, S. Miller, and Z. Sroczynski, Scalar glueball and meson spectroscopy in unquenched lattice qcd with improved staggered quarks, PoS LAT2005 (2006) 027, hep-lat/0510066].

[27] S. Prelovsek, Effects of staggered fermions and mixed actions on the scalar correlator, Phys. Rev. D73 (2006) 014506, hep-lat/0510080].

[28] C. Bernard, C. DeTar, Z. Fu, and S. Prelovsek, Scalar meson spectroscopy with lattice staggered fermions, arXiv:0707.2402 [hep-lat]

[29] Particle Data Group Collaboration, W. M. Yao et. al., Review of particle physics, J. Phys. G33 (2006) 1-1232.

[30] CLQCD Collaboration, Y. Chen, C. Liu, Y. Liu, J. Ma, and J. Zhang, Radially excited states of 1p charmonium and x(3872), hep-lat/0701021.

[31] N. Mathur et. al., A study of pentaquarks on the lattice with overlap fermions, Phys. Rev. D70 (2004) 074508, [hep-ph/0406196].

[32] M. R. Pennington, Location, correlation, radiation: where is the $\sigma$, what is its structure and what is its coupling to photons?, Mod. Phys. Lett. A22 (2007) 1439-1458, [arXiv: 0705.3314 [hep-ph]].

[33] J. J. Dudek and R. G. Edwards, Two photon decays of charmonia from lattice qcd, Phys. Rev. Lett. 97 (2006) 172001, [hep-ph/0607140].

[34] S. Narison, Qcd tests of the puzzling scalar mesons, Phys. Rev. D73 (2006) 114024, [hep-ph/0512256]. 
[35] M. Diehl and G. Hiller, New ways to explore factorization in b decays, JHEP 06 (2001) 067, [hep-ph/0105194].

[36] A. Holl, A. Krassnigg, and C. D. Roberts, Pseudoscalar meson radial excitations, Phys. Rev. C70 (2004) 042203, [nucl-th/0406030].

[37] UKQCD Collaboration, C. McNeile and C. Michael, The decay constant of the first excited pion from lattice qcd, Phys. Lett. B642 (2006) 244-247, hep-lat/0607032].

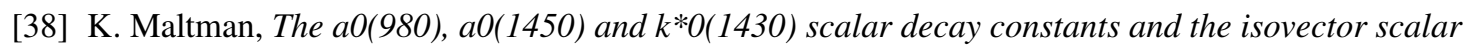
spectrum, Phys. Lett. B462 (1999) 14-22, [hep-ph/9906267].

[39] C. M. Shakin and H. Wang, Calculation of the decay constants of scalar mesons, Phys. Rev. D63 (2001) 074017

[40] H.-Y. Cheng, C.-K. Chua, and K.-C. Yang, Charmless hadronic b decays involving scalar mesons: Implications to the nature of light scalar mesons, Phys. Rev. D73 (2006) 014017, [hep-ph/0508104].

[41] UKQCD Collaboration, G. Herdoiza, C. McNeile, and C. Michael, Decay constants of p-wave heavy-light mesons from unquenched lattice qcd, Phys. Rev. D74 (2006) 014510, [hep-lat/0604001].

[42] C. J. Morningstar and M. J. Peardon, The glueball spectrum from an anisotropic lattice study, Phys. Rev. D60 (1999) 034509, hep-lat/9901004.

[43] UKQCD Collaboration, A. Hart and M. Teper, On the glueball spectrum in o(a)-improved lattice qcd, Phys. Rev. D65 (2002) 034502, [hep-lat/0108022].

[44] I. Caprini, G. Colangelo, and H. Leutwyler, Mass and width of the lowest resonance in qcd, Phys. Rev. Lett. 96 (2006) 132001, [hep-ph/0512364].

[45] Y. Chen et. al., Glueball spectrum and matrix elements on anisotropic lattices, Phys. Rev. D73 (2006) 014516, [hep-lat/0510074].

[46] W.-J. Lee and D. Weingarten, Scalar quarkonium masses and mixing with the lightest scalar glueball, Phys. Rev. D61 (2000) 014015, [hep-lat/9910008].

[47] UKQCD Collaboration, C. McNeile and C. Michael, Mixing of scalar glueballs and flavour-singlet scalar mesons, Phys. Rev. D63 (2001) 114503, hep-lat/0010019.

[48] TXL Collaboration, G. S. Bali et. al., Static potentials and glueball masses from qcd simulations with wilson sea quarks, Phys. Rev. D62 (2000) 054503, [hep-lat/0003012].

[49] SCALAR Collaboration, T. Kunihiro et. al., Scalar mesons in lattice qcd, Phys. Rev. D70 (2004) 034504, [hep-ph/0310312].

[50] UKQCD Collaboration, A. Hart, C. McNeile, C. Michael, and J. Pickavance, A lattice study of the masses of singlet 0++ mesons, Phys. Rev. D74 (2006) 114504, [hep-lat/0608026].

[51] Y. Chen et. al., The sequential empirical bayes method: An adaptive constrained-curve fitting algorithm for lattice qcd, hep-lat/0405001.

[52] N. Mathur et. al., Roper resonance and s(11)(1535) from lattice qcd, Phys. Lett. B605 (2005) 137-143, [hep-ph/0306199].

[53] B. G. Lasscock et. al., Even parity excitations of the nucleon in lattice qcd, arXiv: 0705.0861 [hep-lat]. 
[54] H. Suganuma, K. Tsumura, N. Ishii, and F. Okiharu, Tetra-quark resonances in lattice qcd, arXiv:0707.3309 [hep-lat].

[55] C. Urbach, Lattice qcd with two light wilson quarks and maximally twisted mass, PoS(LAT2007)022 (2007).

[56] P. Boyle, 2+1 flavour domain wall fermion simulations by the rbc and ukqcd collaborations, PoS(LAT2007)005 (2007).

[57] A. Gray et. al., The upsilon spectrum and m(b) from full lattice qcd, Phys. Rev. D72 (2005) 094507, [hep-lat/0507013].

[58] S. Aoki, Unquenched qcd simulation results, Nucl. Phys. Proc. Suppl. 94 (2001) 3-18, [hep-lat/0011074].

[59] K. Jansen, Actions for dynamical fermion simulations: Are we ready to go?, Nucl. Phys. Proc. Suppl. 129 (2004) 3-16, hep-lat/0311039.

[60] C. T. H. Davies, G. P. Lepage, F. Niedermayer, and D. Toussaint, The quenched continuum limit, Nucl. Phys. Proc. Suppl. 140 (2005) 261-263, hep-lat/0409039.

[61] G. Colangelo, S. Durr, and C. Haefeli, Finite volume effects for meson masses and decay constants, Nucl. Phys. B721 (2005) 136-174, [hep-lat/0503014].

[62] C. Bernard, Status of the milc light pseudoscalar meson project, PoS(LAT2007)090 (2007).

[63] S. R. Beane, P. F. Bedaque, K. Orginos, and M. J. Savage, $f(k) / f(p i)$ in full qcd with domain wall valence quarks, Phys. Rev. D75 (2007) 094501, [hep-lat/ 0606023 ].

[64] HPQCD Collaboration, E. Follana, C. T. H. Davies, G. P. Lepage, and J. Shigemitsu, High precision determination of the pi, $k, d$ and $d s$ decay constants from lattice qcd, arXiv:0 706.1726 [hep-lat].

[65] L. Del Debbio, L. Giusti, M. Luscher, R. Petronzio, and N. Tantalo, Qcd with light wilson quarks on fine lattices. $i$ : First experiences and physics results, JHEP 02 (2007) 056, hep-lat/ 0610059.

[66] L. Del Debbio, L. Giusti, M. Luscher, R. Petronzio, and N. Tantalo, Qcd with light wilson quarks on fine lattices. ii: Dd-hmc simulations and data analysis, JHEP 02 (2007) 082, hep-lat/ 0701009 .

[67] M. Gockeler et. al., Simulating at realistic quark masses: Pseudoscalar decay constants and chiral logarithms, PoS LAT2006 (2006) 179, [hep-lat/0610066].

[68] ETM Collaboration, P. Boucaud et. al., Dynamical twisted mass fermions with light quarks, Phys. Lett. B650 (2007) 304-311, hep-lat/0701012.

[69] C. Aubin et. al., Charmed meson decay constants in three-flavor lattice qcd, Phys. Rev. Lett. 95 (2005) 122002, [hep-lat/0506030].

[70] ALPHA Collaboration, J. Heitger, R. Sommer, and H. Wittig, Effective chiral lagrangians and lattice qcd, Nucl. Phys. B588 (2000) 377-399, hep-lat/0006026.

[71] UKQCD Collaboration, A. C. Irving, C. McNeile, C. Michael, K. J. Sharkey, and H. Wittig, Is the up-quark massless?, Phys. Lett. B518 (2001) 243-251, hep-lat/0107023.

[72] RBC and UKQCD Collaboration, C. Allton et. al., 2+1 flavor domain wall qcd on a (2-fm)**3 lattice: Light meson spectroscopy with $l(s)=16$, Phys. Rev. D76 (2007) 014504, hep-lat/0701013]. 
[73] UKQCD Collaboration, C. McNeile and C. Michael, Hadronic decay of a vector meson from the lattice, Phys. Lett. B556 (2003) 177-184, hep-lat/0212020].

[74] CP-PACS Collaboration, S. Aoki et. al., Lattice qcd calculation of the $\rho$ meson decay width, arXiv:0708.3705 [hep-lat].

[75] D. B. Leinweber, A. W. Thomas, K. Tsushima, and S. V. Wright, Chiral behaviour of the rho meson in lattice qcd, Phys. Rev. D64 (2001) 094502, [hep-lat/0104013].

[76] C. R. Allton, W. Armour, D. B. Leinweber, A. W. Thomas, and R. D. Young, Chiral and continuum extrapolation of partially-quenched lattice results, Phys. Lett. B628 (2005) 125-130, [hep-lat/0504022].

[77] P. C. Bruns and U.-G. Meissner, Infrared regularization for spin-1 fields, Eur. Phys. J. C40 (2005) 97-119, [hep-ph/0411223].

[78] G. Schierholz, Probing the chiral limit with clover fermions $i$ : The meson sector, PoS(LAT2007)133 (2007).

[79] W. J. Marciano, Precise determination of $|v(u s)|$ from lattice calculations of pseudoscalar decay constants, Phys. Rev. Lett. 93 (2004) 231803, [hep-ph/ 0402299 ].

[80] HPQCD Collaboration, C. T. H. Davies et. al., High-precision lattice qcd confronts experiment, Phys. Rev. Lett. 92 (2004) 022001, [hep-lat/0304004].

[81] T. A. DeGrand, Lattice qcd spectroscopy: progress and prospects, Nucl. Phys. Proc. Suppl. 20 (1991) 353-361.

[82] R. Sommer, A new way to set the energy scale in lattice gauge theories and its applications to the static force and alpha-s in su(2) yang-mills theory, Nucl. Phys. B411 (1994) 839-854, [hep-lat/9310022].

[83] C. J. Morningstar and M. J. Peardon, Efficient glueball simulations on anisotropic lattices, Phys. Rev. D56 (1997) 4043-4061, hep-lat/9704011.

[84] JLQCD Collaboration, S. Aoki et. al., Light hadron spectroscopy with two flavors of o(a)-improved dynamical quarks, Phys. Rev. D68 (2003) 054502, [hep-lat/0212039].

[85] M. Gockeler et. al., A determination of the lambda parameter from full lattice qcd, Phys. Rev. D73 (2006) 014513, [hep-ph/0502212].

[86] ALPHA Collaboration, R. Sommer et. al., Large cutoff effects of dynamical wilson fermions, Nucl. Phys. Proc. Suppl. 129 (2004) 405-407, hep-lat/0309171].

[87] T. Bhattacharya, R. Gupta, W. Lee, S. R. Sharpe, and J. M. S. Wu, Improved bilinears in lattice qcd with non-degenerate quarks, Phys. Rev. D73 (2006) 034504, [hep-lat/ 0511014 ].

[88] M. Li, The static quark potential for dynamical domain wall fermion simulations, PoS LAT2006 (2006) 183, [hep-lat/0610106.

[89] QCDSF-UKQCD Collaboration, S. Booth et. al., Determination of lambda(ms-bar) from quenched and $n(f)=2$ dynamical qcd, Phys. Lett. B519 (2001) 229-237, [hep-lat/0103023].

[90] UKQCD Collaboration, A. C. Irving et. al., Tuning actions and observables in lattice qcd, Phys. Rev. D58 (1998) 114504, hep-lat/9807015].

[91] C. Bernard et. al., Lattice calculation of 1-+ hybrid mesons with improved kogut-susskind fermions, Phys. Rev. D68 (2003) 074505, [hep-lat/0301024].

[92] M. Luscher, Signatures of unstable particles in finite volume, Nucl. Phys. B364 (1991) 237-254. 